\title{
48. Release of Endogenous Glutamate from the Neuromuscular Junction of the Crayfish
}

\author{
By Akira TAKeuchi, Kayoko Onodera, and Reiko KaWAgoE \\ Department of Physiology, School of Medicine, Juntendo Univ., \\ Hongo, Tokyo 113 \\ (Communicated by Yasuji Katsuki, M. J. A., April 12, 1980)
}

Several lines of evidence suggest that L-glutamic acid acts as the excitatory transmitter at the crustacean neuromuscular junction. ${ }^{1-4)}$ Among them are the facts that: a) The glutamate sensitivity is localized at the excitatory synapse; b) the action of L-glutamate is specifically strong among related substances; c) the time course of the action of L-glutamate is comparable to that of the excitatory postsynaptic potential (e.p.s.p.) and d) the ionic mechanism of the postsynaptic membrane for L-glutamate is identical with that for the e.p.s.p. However, a crucial experiment for this hypothesis: The detection of the release of endogenous glutamate upon nerve stimulation has not been conclusive. This is largely due to a substantial and uncontrollable spontaneous release of glutamate. ${ }^{5)}$ Since glutamic acid is contained in many types of tissues, these tissues may leake glutamate. In the present experiment, therefore, muscle fibres were removed from the animal and thoroughly washed, and glutamate content in the bath solution was measured with the mass fragmentography using a gaschromatograph-mass spectrometer (GC-MS). This method is highly sensitive and specific. It was found that a significant amount of glutamate was released by nerve stimulation.

Methods. Ventral superficial muscle was isolated from abdominal flexor of the crayfish (Cambarus clarkii) together with innervating nerve fibres. This muscle forms a sheet which is made of a thin layer of a few muscle fibres' thick. ${ }^{6}$ ) When viewed with Nomarski optics, the axons terminated at many points on the muscle fibre with clusters of bouton-like structures. The e.p.s.p. could be recorded at these boutons with an extracellular microelectrode, and the sensitivity to the iontophoretically applied glutamate was localized at these boutons as observed in the opener muscle of the dactyl in the walking leg. ${ }^{7)}$

The preparation was placed in a small bath of about $0.1 \mathrm{ml}$ in volume, and fixed with fine needles penetrating both ends of the tendon. Chilled Harreveld's solution was pumped into the bath at the rate of $0.75 \mathrm{ml} / \mathrm{min}$ and removed with a hypodermic needle connected to a suction pump. After washing for 30-60 min, the bath solution was removed with a suction pump and $100 \mu$ of Harreveld's solution was 
put into the bath with a pipett. In ordeter to prevent a possible uptake of glutamate to the surrounding tissues, the bath solution was cooled to $9{ }^{\circ} \mathrm{C}$ with a thermoelement. After $10 \mathrm{~min}$, the solution was removed with a syringe and stored in a prechilled test tube. Each time, between introducing the solution with a pipette, the preparation was washed for a minute or two with cold Harreveld's solution at the rate of $0.75 \mathrm{ml} / \mathrm{min}$. The nerve stimulation was applied for 5-8 min with a pair of silver wires, and the e.p.s.p. was recorded with an intracellular microelectrode during this period.

From each test tube, 50 or $75 \mu$ l of the solution was sampled and deuterium-labeled L-glutamate (2d-L-glutamic acid, Aldrich Chemicals) (50 pmole) was added as an internal standard. Also included in each experiment were samples containing $0,5,10,20,40$ and 100 pmole of L-glutamate plus internal standard in Harreveld's solution which had not been in contact with muscle fibres. The samples were evaporated to dryness overnight at room temperature under vacuo.

Details of the amino acid analysis using GC-MS (Finnigan Type 4000) will be reported elsewhere (Murayama, Morishita, Shindo and Ohta, unpublished). Pentafluoropropionyl (PFP) glutamate methyl ester was prepared by a modified method of Caprioli, Seifer and Sutherland. ${ }^{8)}$ The mass spectrum of PFP glutamate methyl ester gave major peaks at $m / e$ 322, 290 and 262. Among these peaks, $m / e 262$ was the strongest and was used for the present analysis. The glutamate concentration was calculated from the ratio of the area under mass fragmentogram of glutamate to deuterium-labeled glutamate $(\mathrm{m} / \mathrm{e}$ $262 / 263)$. With this method 1 pmole/100 $\mu$ l of glutamate was measured.

Results. The solid columns in Fig. 1 show the glutamate content of control rest periods. The background release of glutamate was relatively stable and the average value was $20.4 \pm 0.75 \mathrm{pmole} / 10 \mathrm{~min}$ $(n=200) \quad$ (mean \pm S.E.M). Stippled column in Fig. $1 \mathrm{~A}$ shows the glutamate content, when the nerve was stimulated at $25 \mathrm{~Hz}$ for $7 \mathrm{~min}$. In Fig. $1 \mathrm{~B}$ stimulation was applied twice at $20 \mathrm{~Hz}$. Although there was a considerable variation, a significant increase in the glutamate content was produced by nerve stimulation $(\mathrm{P}<0.01)$. Average amount of glutamate released in excess of the background (net release) was $20.3 \pm 2.4$ pmole $(n=11)$ with stimulation at $20 \mathrm{~Hz}$ and $11.9 \pm 3.0$ pmole $(n=6)$ at $10 \mathrm{~Hz}$.

Fig. 2 shows the effect of Ca concentration on the glutamate release produced by $20 \mathrm{~Hz}$ stimulation. During the period indicated by the bar the Ca concentration was reduced to $10 \%$ of the normal solution containing $5.3 \mathrm{mM} \mathrm{Mg}$. Sample records of the e.p.s.p. before, during and after changing the $\mathrm{Ca}$ concentration are shown in inset. The release of glutamate by nerve stimulation was decreased in low-Ca 


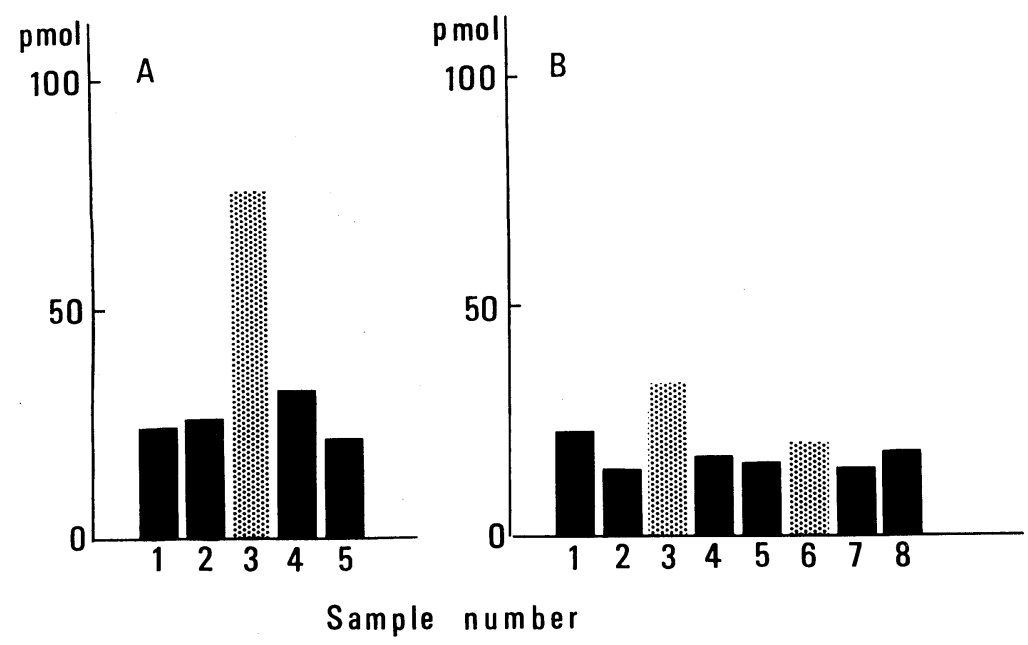

Fig. 1. Release of glutamate upon nerve stimulation. Each column represents glutamate content for a sampling period of $10 \mathrm{~min}$. A: The nerve stimulation was applied at $25 \mathrm{~Hz}$ for $7 \mathrm{~min}$ and the glutamate content in this solution is shown in the stippled column. B: Stimulation was applied twice at $20 \mathrm{~Hz}$ for $7 \mathrm{~min}$.

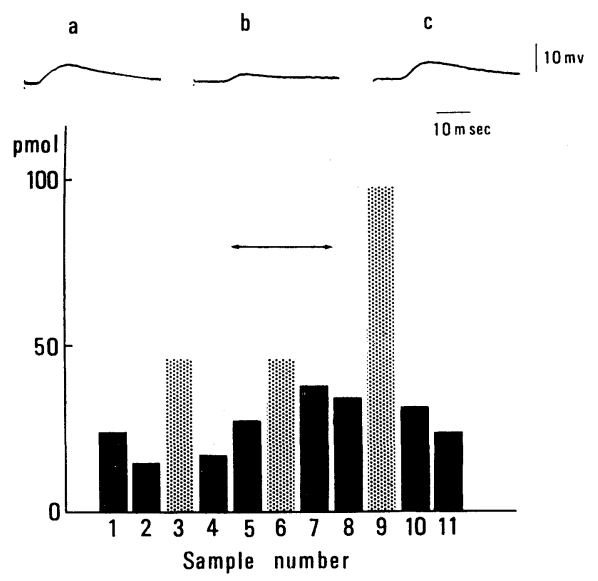

Fig. 2 Effect of low-Ca solution on the glutamate release. During the period indicated by the bar the $\mathrm{Ca}$ concentration was decreased to $10 \%$ of the normal solution containing $5.3 \mathrm{mM} \mathrm{Mg}$. The e.p.s.p.'s are shown in inset which were produced at $20 \mathrm{~Hz}$ in low Ca medium, and in normal solutions before and after changing the Ca concentration.

solution. Average value of net release produced by $20 \mathrm{~Hz}$ stimulation in normal solution was $21.6 \pm 3.19$ pmole $(n=6)$ before changing the Ca concentration. In the low-Ca solution, the net release was $8.8 \pm 3.49$ pmole $(n=6) \quad(\mathrm{P}<0.025$ when compared with control in normal solution). And after returning to normal solution, it was $31.7 \pm 2.59$ pmole 
$(n=3)$. In low Ca solution containing $\mathrm{Mg}$ the background release showed a tendency to increase. Since spontaneous e.p.s.p. often appeared repetitively, the increase in the background release may be attributable to spontaneous e.p.s.p. The average value of background in low-Ca solution was $28.4 \pm 2.28$ pmole $/ 10 \mathrm{~min}(n=33)(\mathrm{P}<0.01$ when compared with control).

Discussion. In conjunction with findings in previous experiments, ${ }^{1,3)-5)}$ the present results give strong support to the hypothesis that L-glutamic acid is an excitatory transmitter at the crayfish neuromuscular junction.

Acknowledgements. We thank K. Murayama, N. Shindo, R. Morishita and K. Ohta of the Central Laboratory of Medical Sciences, Juntendo University for their invaluable help in the glutamate analysis. These experiments were supported in part by grants from the Ministry of Education, Science and Culture.

\section{References}

1) Takeuchi, A., and Takeuchi, N.: Advances in Biophysics, 3, 45-95 (1972).

2) Gerschenfeld, H. M.: Physiol. Rev., 53, 1-119 (1973).

3) Onodera, K., and Takeuchi, A.: J. Physiol., Lond., 252, 295-318 (1975).

4) —-: J. Physiol., Lond., 255, 669-685 (1976).

5) Kravitz, E. A., Slater, C. R., Takahashi, K., Bownds, M. D., and Grossfeld, R. M.: Excitatory Synaptic Mechanisms (eds. Andersen, P., and Jansen, J. K. S.). Universitetsforlaget, Oslo, pp. 84-93 (1970).

6) Kennedy, D., and Takeda, K.: J. exp. Biol., 43, 229-246 (1965).

7) Onodera, K., and Takeuchi, A.: J. Physiol., Lond. (in press).

8) Caprioli, R. M., Seifert Jr., W. E., and Sutherland, D. E.: Biochem. Biophys. Res. Comm., 55, 67-75 (1973). 